\title{
Comparison of four diagnostic criteria for invasive pulmonary aspergillosis - a diagnostic accuracy study in critically ill patients
}

\author{
Maria Schroeder ( $\square$ m.metschke@uke.de) \\ University Medical Center Hamburg-Eppendorf \\ Melanie Giese \\ University Medical Center Hamburg-Eppendorf \\ Charles Wijaya \\ University Medical Center Hamburg-Eppendorf
}

Sarah Winterland

University Medical Center Hamburg-Eppendorf

Annika Nuechtern

University Medical Center Hamburg-Eppendorf

Joern Grensemann

University Medical Center Hamburg-Eppendorf

Hanna Matthews

University Medical Center Hamburg-Eppendorf

Dominic Wichmann

University Medical Center Hamburg-Eppendorf

Johannes Stamm

University Medical Center Hamburg-Eppendorf

\section{Holger Rohde}

University Medical Center Hamburg-Eppendorf

\section{Martin Christner}

University Medical Center Hamburg-Eppendorf

\section{Ann-Kathrin Ozga}

University Medical Center Hamburg-Eppendorf

\section{Stefan Steurer}

University Medical Center Hamburg-Eppendorf

Axel Heinemann

University Medical Center Hamburg-Eppendorf

\section{Marcel Simon}

University Medical Center Hamburg-Eppendorf

\section{Marlene Fischer}

University Medical Center Hamburg-Eppendorf 


\section{Stefan Kluge}

University Medical Center Hamburg-Eppendorf

\section{Research Article}

Keywords: Aspergillus, invasive pulmonary aspergillosis, mycosis, critical care, ICU, diagnostic algorithm, influenza, COVID-19, CAPA, antifungal treatment

Posted Date: March 7th, 2022

DOI: https://doi.org/10.21203/rs.3.rs-1298483/v2

License: (c) (i) This work is licensed under a Creative Commons Attribution 4.0 International License. Read Full License 


\section{Abstract}

\section{BACKGROUND:}

In the absence of lung biopsy there are various algorithms for the diagnosis of invasive pulmonary aspergillosis (IPA) in intensive care unit (ICU) patients that rely on clinical signs, underlying conditions, radiological features, and mycology. The aim of the present study was to compare four diagnostic algorithms in their ability to differentiate between probable IPA (i.e. requiring treatment) and colonization in critically ill patients. In addition, we determined their diagnostic accuracy to detect proven IPA.

\section{METHODS:}

For this diagnostic accuracy study, we included a mixed ICU population with a positive Aspergillus culture from respiratory secretions that were treated in a German quarternary care hospital between 2005 and 2020 . Four different diagnostic algorithms were applied to all patients. We compared agreement among the four algorithms to discriminate probable IPA from colonization. In a subgroup of patients with lung tissue histopathology available we determined the sensitivity and specificity of the single algorithms to detect proven IPA.

\section{RESULTS:}

A total number of 684 critically ill patients were included. The mean age of the study population was 63 years (IQR 52-73). Sixty-nine percent $(n=472)$ were medical and 31\% $(n=212)$ were surgical ICU patients. Overall, $79 \%$ ( $n=543$ ) of patients fulfilled the criteria for probable IPA according to at least one diagnostic algorithm. Only $4 \%$ of patients $(n=29)$ fulfilled the criteria for probable IPA according to all four algorithms. Agreement among the four diagnostic criteria was low (Cohen's kappa 0.07-0.29).

From 85 patients with histopathological examination of lung tissue $40 \%(n=34)$ had confirmed IPA. The new EORTC/MSGERC ICU working group criteria had high specificity (0.59 [0.41-0.75]) and sensitivity (0.73 [0.590.85]).

\section{CONCLUSIONS:}

In a cohort of mixed ICU patients, the agreement among four algorithms for the diagnosis of IPA was low. The 2021 EORTC/MSGERC ICU working group criteria achieved the highest specificity and sensitivity to detect proven IPA. Although improved by the latest diagnostic criteria, the discrimination of invasive fungal infection from Aspergillus colonization in critically ill patients remains challenging and may require further optimization.

\section{Background}

Invasive pulmonary aspergillosis (IPA) has become an emerging disease in critically ill patients without classical risk factors, including neutropenia, stem cell or solid organ transplantation [1]. 
During the ongoing SARS-CoV-2 pandemic, IPA has been observed more frequently in patients with coronavirus disease 2019 (COVID-19) [2-4]. In addition, severe influenza has been identified as a risk factor for IPA $[5,6]$. Importantly, reports on the incidence of IPA in critically ill patients vary considerably ranging between $0.3 \%$ and $19 \%$ [7-9]. For the diagnosis of proven IPA, according to the EORTC criteria, the histopathological assessment of lung tissue is mandatory. Therefore, the confirmation of suspected IPA is difficult in critically ill patients due to the high risks of invasive sampling for histological examination [10, 11]. In addition to the diagnostic criteria recommended by the European Organization for Research and Treatment of Cancer and the Mycoses Study Group Education and Research Consortium (EORTC/MSGERC) for hemato-oncological patients, several research groups have introduced alternative diagnostic algorithms for IPA in critically ill patients. In the absence of histopathological specimen or if a biopsy material is not available, the presence of clinical symptoms, imaging characteristics, microbiological features, and underlying conditions allow for a diagnosis of probable IPA $[5,9,12,13]$. Yet, the challenge in IPA diagnosis is the differentiation between IPA as an infectious disease and Aspergillus colonization. Therefore, the major aim of all diagnostic algorithms is to distinguish probable IPA requiring antifungal treatment from colonization without the necessity of antifungal medication. The aim of this study was to compare four different algorithms for the diagnosis of IPA in critically ill adults $[5,9,12,14]$. Therefore, we retrospectively reviewed medical records and mycological findings from 684 patients over a 15-year period. In addition, we analyzed diagnostic agreement between single algorithms and we identified proven IPA confirmed by histopathological analysis in a subgroup of patients.

\section{Methods}

\section{Study design and participants}

This diagnostic accuracy study includes all critically ill patients with a positive Aspergillus culture who were admitted to the Department of Intensive Care Medicine at the University Medical Center Hamburg-Eppendorf (UKE) between February $26^{\text {th }}, 2005$ and December $31^{\text {st }}, 2020$. A follow-up survey to determine mortality (90 days) was conducted through March $31^{\text {st }}, 2021$.

\section{Ethics approval and consent to participate}

This study was approved by the ethics committee at the Hamburg State Chamber of Physicians (registration no.: WF 054/11). Retrospective data collection and analysis was conducted in accordance with local government law (HmbKHG. §12) without the requirement for informed consent.

\section{Setting}

The University Medical Center Hamburg-Eppendorf is a quarternary care hospital in Northern Germany. The Department of Intensive Care Medicine includes 12 multidisciplinary intensive care units (ICU) with a total of 140 ICU beds. The department serves all specialties of adult intensive care medicine including solid organ and bone marrow transplantation as well as extracorporeal membrane oxygenation (ECMO) for ARDS and cardiogenic shock. 


\section{Data collection}

The following demographic and clinical variables were collected from the electronic patient data management system (PDMS, Intensive Care Manager V10 and ICMiq V1.3, Drägerwerk, Lübeck, Germany): age, sex, body mass index, route of nutrition, admission diagnosis, comorbidities, host factors, and medication. We collected microbiological results including Aspergillus spp., source of sampling (tracheal secretion, bronchial washing, bronchoalveolar lavage [BAL]), Aspergillus galactomannan enzyme immunoassay in BAL and serum, and ß-D-glucan. In addition, we obtained polymerase chain reaction (PCR) results for SARS-CoV-2 and influenza virus. Acute Physiology and Chronic Health (APACHE II) score on admission, Sequential Organ Failure Assessment (SOFA) score and Simplified Acute Physiology Score II (SAPS II) on the day of first Aspergillus culture finding were calculated. White blood cells, C-reactive protein, body temperature, macroscopic bronchoscopy findings, chest X-ray or computed tomography, and results from histopathological analysis were retrieved. Furthermore, we documented details on critical care management including ventilation settings, requirement of extracorporeal membrane oxygenation, antifungal treatment, length of ICU and hospital stay. Survival status at 90 days was obtained from medical records, telephone interviews with patients, next of kin or primary care physicians, or mortality statistics from the Hamburg registry office.

\section{Diagnostic criteria}

We selected four diagnostic algorithms that were developed or modified for the diagnosis of IPA in critically ill patients: (1) a clinical algorithm for intensive care patients by Blot et al. published in 2012 (AspICU) (subsequently referred to as AspICU*2012), (2) the algorithms published by Schauwvlieghe et al. in 2018 [5] (subsequently referred to as modified AspICU*2018) and (3) Loughlin et al. in 2020 (subsequently referred to as modified AspICU*2020) [9]. (4) Last, we applied the EORTC/MSGERC ICU Working Group criteria published by Bassetti et al. (subsequently referred to as EORTC/MSGERC ICU*2021) to our dataset [12]. All diagnostic criteria evaluate clinical, radiological, and microbiological features. The detailed diagnostic criteria are listed in Additional File 1. We applied the criteria from the EORTC Mycoses Study Group Education and Research Consortium to our dataset without analyzing diagnostic agreement [15].

\section{Microbiological assays}

For Aspergillus culture, 5-10 $\mathrm{ml}$ of respiratory secretions fluid were centrifuged at $3000 \mathrm{G}$. The resulting pellet was re-suspended in $1 \mathrm{ml}$ supernatant and 50-100 $\mu$ l were inoculated onto Sabouraud dextrose-agar with gentamicin and chloramphenicol (Oxoid, Basingstoke, UK). Plates were incubated at $30{ }^{\circ} \mathrm{C}$ for 14 days and inspected regularly. Culture isolates were identified by matrix-assisted laser desorption ionization timeof-flight mass spectrometry fingerprinting or internal transcribed spacer sequencing as required.

Detection of Aspergillus galactomannan in BAL fluid and serum samples was performed using the Platelia Aspergillus enzyme immunoassay (Bio-Rad Laboratories, Munich, Germany) following the manufacturer's instructions. Tests were considered positive at a cut off index $\geq 0.5$ using single-sample aliquots. 
Histopathological examination of lung tissue was performed after hematoxylin-eosin and Grocott methenamine silver staining. Tissue was screened for hyphae, typical filaments and mycelium.

\section{Statistical analysis}

Continuous variables are expressed as median with $1^{\text {st }}$ to $3^{\text {rd }}$ quartiles. Categorical variables are given as absolute and relative numbers. Diagnostic agreement was calculated with Cohen's kappa and categorized values $<0$ as indicating no agreement, $0-0.40$ as poor, $0.41-0.60$ as moderate, $0.61-0.80$ as substantial, and $0.81-1$ as almost perfect agreement. A subgroup analysis was performed for immunocompromised patients. In addition, we performed a sensitivity analysis for patients without viral pneumonia caused by SARS-CoV-2 or influenza. The sensitivity and specificity to identify proven IPA was calculated in a subgroup of patients, for whom lung tissue was available.

Statistical analyses were performed with SPSS ${ }^{\circledR}$ (IBM SPSS Statistics for Windows, Version 27.0. Armonk, NY, USA). The Venn diagram for probable IPA and agreement plots were designed with R® version 4.0.3. Specificity and sensitivity with $95 \%$ confidence intervals were calculated with $\mathrm{R} \circledast$ version 4.0.3.

\section{Results}

\section{Study population}

During the study period from 2005 to 2020 , we identified 684 patients with a positive Aspergillus culture from respiratory secretions. The flow of participants is shown in Figure 1. The median age was 63 years (IQR 52-73). More than two thirds of patients $(n=472,69 \%)$ were admitted to the ICU due to medical conditions with a median length of stay of 18 days (IQR 9-36). The vast majority ( $n=630,92 \%$ ) of all study participants were mechanically ventilated; 77 (11\%) required ECMO support. The ICU mortality was $54 \%$ $(\mathrm{n}=369)$. At 90 days after first Aspergillus detection 402 patients (59\%) deceased (Table 1). Twenty-eight patients (4\%) suffered from severe influenza and $13(2 \%)$ were treated for COVID-19.

\section{Table 1}




\section{Demographics}

\begin{tabular}{|c|c|}
\hline Sex (female) & $248(36)$ \\
\hline Age (years) & $63(52-73)$ \\
\hline Body mass index $\left(\mathrm{kg}^{\star} \mathrm{m}^{-2}\right)$ & $25(22-28)$ \\
\hline \multicolumn{2}{|l|}{ Baseline characteristics } \\
\hline Medical admission & $472(69)$ \\
\hline Surgical admission & $212(31)$ \\
\hline APACHE II & $21(16-26)$ \\
\hline SAPS II & $49(39-64)$ \\
\hline SOFA & $11(8-14)$ \\
\hline \multicolumn{2}{|l|}{ ICU Stay } \\
\hline \multicolumn{2}{|l|}{ Ventilation } \\
\hline No mechanical ventilation & $20(3)$ \\
\hline NIV & $34(5)$ \\
\hline Mechanical ventilation & $441(65)$ \\
\hline NIV and MV & $189(28)$ \\
\hline ECMO support & $77(11)$ \\
\hline Antifungal treatment & $445(66)$ \\
\hline Azole (Voriconazole or Isavuconazole) & $329(48)$ \\
\hline Echinocandin (Caspofungin or Anidulafungin) & $168(25)$ \\
\hline Liposomal Amphotericin B & $134(20)$ \\
\hline Change of antifungal agent & $163(24)$ \\
\hline Combination therapy & $89(13)$ \\
\hline ICU LOS (days) & $18(9-36)$ \\
\hline \multicolumn{2}{|l|}{ Outcome } \\
\hline ICU mortality & $369(54)$ \\
\hline 28-day mortality & $329(49)$ \\
\hline 90-day mortality & $402(59)$ \\
\hline
\end{tabular}

Baseline demographic and clinical characteristics, critical care management, and outcome data from 684 patients with positive culture of Aspergillus spp. in respiratory secretions. Continuous variables are given as 
median ( $1^{\text {st }}$ to $3^{\text {rd }}$ quartile), categorical variables are given as $\mathrm{n}(\%)$.

APACHE II: Acute Physiology and Chronic Health Evaluation Score, ECMO: Extracorporeal membrane oxygenation, ICU: Intensive Care Unit, LOS: Length of stay, MV: Mechanical ventilation, NIV: Non-invasive ventilation, SAPS II: Simplified Acute Physiology Score II, SOFA: Sequential Organ Failure Assessment Score

Diagnostic agreement for probable IPA

In addition to microbiological, clinical, and radiological findings the AspICU*2012 and EORTC/MSGERC ICU*2021 criteria require host-related risk factors for the definition of probable IPA. The diagnostic findings stratified by the four algorithms are shown in Table 2.

According to the AspICU*2012 criteria [14], 419 (62\%) patients had probable (= putative) IPA. A total of 419 (62\%) patients fulfilled the criteria for probable IPA according to the modified AspICU*2018 criteria [5]

(Figure 1). Importantly, probable IPA based on the AspICU*2012 criteria did not apply to the same individuals than probable IPA based on the modified AspICU*2018 algorithm (Figure 2 and 3 ). The modified AspICU*2020 criteria and the EORTC/MSGERC ICU*2021 criteria for probable IPA were fulfilled by 116 (17\%) and $118(17 \%)$ patients, respectively.

The distribution of probable IPA according to single algorithms and the overlap among the four diagnostic criteria is presented in Figure 2. AspICU $* 2012$ classified $24 \%(n=101 / 419)$ of the patients as probable, with no other diagnostic algorithm classifying them as such. In addition, $39 \%(n=163 / 419)$ of the patients classified as probable IPA by the AspICU* 2012 criteria also fulfilled the criteria for probable IPA by the modified AspICU*2018 criteria. Fifty-seven (14\%) patients had probable IPA according to AspICU*2012, modified AspICU*2018, and modified AspICU*2020. Also 57 patients (14\%), but not the same individuals, had probable IPA based on AspICU*2012, modified AspICU*2018, and EORTC/MSGERC ICU*2021. 


\begin{tabular}{|c|c|c|c|}
\hline \multirow{2}{*}{$\begin{array}{l}\text { AsplCU } \\
\star 2012\end{array}$} & & $\begin{array}{l}\text { modified } \\
\text { AsplCU*2020 }\end{array}$ & $\begin{array}{l}\text { EORTC/MSGERC } \\
\text { ICU*2021 }\end{array}$ \\
\hline & $\begin{array}{l}\text { modifıed } \\
\text { AspICU*2018 }\end{array}$ & $n=116$ & $n=118$ \\
\hline & $=419$ & & \\
\hline
\end{tabular}

Microbiology

\begin{tabular}{|c|c|c|c|c|c|}
\hline \multirow[t]{2}{*}{ Aspergillus species } & A. fumigatus & $\begin{array}{l}366 \\
(87)\end{array}$ & $356(85)$ & $101(87)$ & $99(84)$ \\
\hline & $\begin{array}{l}\text { A. non } \\
\text { fumigatus }\end{array}$ & $53(13)$ & $63(15)$ & $15(13)$ & $19(16)$ \\
\hline \multirow[t]{3}{*}{ Microscopy } & mycelium & $25(6)$ & $18(4)$ & $8(7)$ & $10(8)$ \\
\hline & pseudomycelium & $3(1)$ & $6(1)$ & $2(2)$ & $3(3)$ \\
\hline & negative & $\begin{array}{l}391 \\
(93)\end{array}$ & $395(94)$ & $106(91)$ & $105(89)$ \\
\hline \multirow{3}{*}{$\begin{array}{l}\text { Aspergillus } \\
\text { Galactomannan } \\
\text { BAL }\end{array}$} & negative & $70(17)$ & $78(19)$ & $21(18)$ & $12(10)$ \\
\hline & positive & $\begin{array}{l}184 \\
(44)\end{array}$ & $193(46)$ & $57(49)$ & $66(56)$ \\
\hline & not performed & $\begin{array}{l}165 \\
(39)\end{array}$ & $148(35)$ & $38(33)$ & $40(34)$ \\
\hline \multicolumn{2}{|c|}{ Aspergillus Galactomannan BAL EIA } & $4(2-4)$ & $4(2-4)$ & $4(3-4)$ & $4(4-4)$ \\
\hline \multirow{3}{*}{$\begin{array}{l}\text { Aspergillus } \\
\text { Galactomannan } \\
\text { serum }\end{array}$} & negative & $\begin{array}{l}111 \\
(26)\end{array}$ & $105(25)$ & $24(21)$ & $29(25)$ \\
\hline & positive & $44(11)$ & $49(12)$ & $19(16)$ & $27(23)$ \\
\hline & not performed & $\begin{array}{l}264 \\
(63)\end{array}$ & $265(63)$ & $73(63)$ & $62(53)$ \\
\hline \multicolumn{2}{|c|}{ Aspergillus Galactomannan serum EIA } & $2(1-4)$ & $2(1-4)$ & $1(1-4)$ & $3(1-5)$ \\
\hline \multicolumn{6}{|l|}{ Clinical signs } \\
\hline \multicolumn{2}{|c|}{ Dyspnea; respiratory failure } & $\begin{array}{l}419 \\
(100)\end{array}$ & $419(100)$ & $116(100)$ & $118(100)$ \\
\hline \multirow{3}{*}{$\begin{array}{l}\text { Bronchoscopy with } \\
\text { Aspergillus specific } \\
\text { lesions }\end{array}$} & none & $\begin{array}{l}343 \\
(82)\end{array}$ & $342(82)$ & $89(77)$ & $64(54)$ \\
\hline & positive & $36(9)$ & $35(8)$ & $18(16)$ & $48(41)$ \\
\hline & $\begin{array}{l}\text { no } \\
\text { bronchoscopy }\end{array}$ & $40(10)$ & $42(10)$ & $9(8)$ & $6(5)$ \\
\hline \multicolumn{2}{|c|}{ purulent tracheal secretion } & $\begin{array}{l}239 \\
(57)\end{array}$ & $251(60)$ & $98(84)$ & $74(63)$ \\
\hline \multicolumn{2}{|c|}{ pulmonary hemorrhage } & $71(17)$ & $77(18)$ & $15(13)$ & $16(14)$ \\
\hline
\end{tabular}


Body temperature $\left({ }^{\circ} \mathrm{C}\right)$

Leukocytes (Mrd/I)

Antibacterial treatment
37 (37- $\quad 37$ (37-38)

38)

12 (7- 12 (8-19)

19)

418

(100)

418 (100)
38 (37-38)

$37(37-38)$

$15(10-21)$

$12(6-20)$

$116(100)$

$118(100)$

\section{Radiology}

Computed

tomography with

Aspergillus specific

lesions none

positive

no imaging
215

(51)

74 (18) $85(20)$

130

(31)

$217(52)$

117 (28)

$58(50)$

20 (17)

419

Pathological x-ray

419
$(100)$

Worsening alveolar infiltrates

154
$(37)$

$27(23)$

$83(70)$

$31(27)$

$15(13)$

Opacites in x-ray

none $\quad 0$

infiltrate

117

(28)

effusion

113

(27)

both

189

(45)

$419(100)$

$116(100)$

$109(92)$

$116(100)$

$51(43)$

$200(48)$

0

0

$9(8)$

157 (37)

$46(40)$

34 (29)

$1(1)$

$13(11)$

$262(63)$

69 (59)

$62(53)$

\section{$\underline{\text { Risc factors }}$}

Host factors (EORTC)

218

(52)

Neutropenia

35 (8) $\quad 32(8)$

$11(9)$

14 (12)

Immunosuppressive treatment

$162 \quad 127(30)$

(39)

Hematological disease

79 (19) $64(15)$

39 (34)

$56(47)$

Chemotherapy

87 (21) $63(15)$

20 (17)

26 (22)

Oncological disease

103
$(25)$

Allogeneic stem cell transplantation

47 (11) $\quad 38(9)$

$10(9)$

14 (12)

Autologous stem cell transplantation

$6(1) \quad 6(1)$

3 (3)

5 (4)

Lung transplantation

$10(2) \quad 9(2)$

$1(1)$

4 (3)

Other SOT

7 (6)

$18(15)$ 


\begin{tabular}{|c|c|c|c|c|}
\hline COPD & $75(18)$ & $68(16)$ & $18(16)$ & $18(15)$ \\
\hline Decompensated liver cirrhosis & $56(13)$ & $48(12)$ & $14(12)$ & $21(18)$ \\
\hline $\begin{array}{l}\text { Prednisolone or its equivalent more } \\
\text { than } 20 \mathrm{mg} / \text { day }\end{array}$ & $\begin{array}{l}351 \\
(84)\end{array}$ & $259(62)$ & $75(65)$ & $93(79)$ \\
\hline HIV & $18(4)$ & $17(4)$ & $3(3)$ & $4(3)$ \\
\hline Severe influenza & $14(3)$ & $27(6)$ & $9(8)$ & $7(6)$ \\
\hline COVID-19 & $8(2)$ & $13(3)$ & $7(6)$ & $5(4)$ \\
\hline Specificity $^{\text {a }}$ & $\begin{array}{l}0.27 \\
(0.15 \\
0.41)\end{array}$ & $\begin{array}{l}0.29(0.17 \\
0.43)\end{array}$ & $\begin{array}{l}0.78(0.63, \\
0.88)\end{array}$ & $0.73(0.59,0.85)$ \\
\hline Sensitivity ${ }^{a}$ & $\begin{array}{l}0.82 \\
(0.65 \\
0.93)\end{array}$ & $\begin{array}{l}0.65(0.46 \\
0.80)\end{array}$ & $\begin{array}{l}0.29(0.15 \\
0.47)\end{array}$ & $0.59(0.41,0.75)$ \\
\hline
\end{tabular}

\section{Table 2}

Probable IPA stratified by the number of patients fulfilling the criteria in each of the four diagnostic algorithms. Continuous variables are given as median ( $1^{\text {st }}$ to $3^{\text {rd }}$ quartile), categorical variables are given as $\mathrm{n}(\%)$. The relevant criteria for one specific diagnostic algorithm are highlighted in blue.

BAL: Bronchoalveolar Lavage, COPD: Chronic obstructive pulmonary disease, COVID-19: Corona Virus Disease 2019, EORTC: European Organisation for Research and Treatment of Cancer, EIA: enzyme-linked immunoassay. HIV: human immunodeficiency virus, SOT: Solid Organ Transplantation

a to detect definite IPA confirmed by histopathological results. (95\% Confidence Interval)

Criteria that are relevant for the diagnosis with the respective algorithm are highlighted in bold.

Overall, $543(79 \%)$ patients were found to have probable IPA with at least one diagnostic tool. In contrast, only 29 (4\%) patients fulfilled the criteria for probable IPA according to all four algorithms (Figure 2).

Diagnostic agreement between the AspICU*2012 and the modified AspICU*2018 criteria was poor with Cohen's kappa of 0.29 (95\% Cl: 0.22, 0.37) (Figure 3). Diagnostic agreement was consistently poor among any one of the four different classifications as reflected by low Cohen's kappa (Figure 3).

\section{Subgroup analysis for histopathological material}

Lung tissue for histopathological examination was available in 85 patients (example presented in Figure 4). A subgroup analysis of biopsy materials showed evidence of confirmed IPA in 40\% ( $n=34 / 85)$ of these patients. Irrespective of the presence of confirmed IPA, we applied the four algorithms to these 85 patients. The AspICU*2012 reached a sensitivity of $0.27(95 \% \mathrm{Cl} 0.15,0.41)$ and a specificity of $0.82(95 \% \mathrm{Cl} 0.65,0$ - 
93), modified AspICU*2018 of $0.29(95 \% \mathrm{Cl} 0.17,0.43)$ and $0.65(95 \% \mathrm{Cl} 0.46,0.80)$, modified AsplCU*2020 of $0.78(95 \% \mathrm{Cl} 0.63,0.88)$ and $0.29(95 \% \mathrm{Cl} 0.15,0.47)$ and EORTC/MSGERC ICU*2021 of $0.73(95 \% \mathrm{Cl}$ $0.59,0,85)$ and $0.59(95 \% \mathrm{Cl}$

$0.14,0.57$ ) respectively (Table 3 ).

\section{Table 3}

\begin{tabular}{|c|c|c|c|c|c|}
\hline & & $\begin{array}{l}\text { AsplCU } \\
\star 2012\end{array}$ & & $\begin{array}{l}\text { modified } \\
\text { AsplCU*2020 }\end{array}$ & $\begin{array}{l}\text { EORTC/MSGERC } \\
\text { ICU*2021 }\end{array}$ \\
\hline & & $n=419$ & $\begin{array}{l}\text { modifled } \\
\text { AsplCU*2018 }\end{array}$ & $n=116$ & $\mathrm{n}=118$ \\
\hline & & & $n=419$ & & \\
\hline Histopathology $n=85$ & Specificity & $\begin{array}{l}0.27 \\
(0.15 \\
0.41)\end{array}$ & $\begin{array}{l}0.29(0.17 \\
0.43)\end{array}$ & $\begin{array}{l}0.78(0.63 \\
0.88)\end{array}$ & $0.73(0.59,0.85)$ \\
\hline & Sensitivity & $\begin{array}{l}0.82 \\
(0.65 \\
0.93)\end{array}$ & $\begin{array}{l}0.65(0.46 \\
0.80)\end{array}$ & $\begin{array}{l}0.29(0.15 \\
0.47)\end{array}$ & $0.59(0.41,0.75)$ \\
\hline Subpopulation analysis & & & & & \\
\hline $\begin{array}{l}\text { Histopathology without } \\
\text { influenza and covid-19 } \\
n=80\end{array}$ & Specificity & $\begin{array}{l}0.25 \\
(0.14 \\
0.40)\end{array}$ & $\begin{array}{l}0.29(0.17 \\
0.44)\end{array}$ & $\begin{array}{l}0.77(0.63, \\
0.88)\end{array}$ & $0.75(0.60,0.86)$ \\
\hline & Sensitivity & $\begin{array}{l}0.84 \\
(0.67 \\
0.95)\end{array}$ & $\begin{array}{l}0.62(0.44 \\
0.79)\end{array}$ & $\begin{array}{l}0.25(0.11 \\
0.43)\end{array}$ & $0.56(0.38,0.74)$ \\
\hline $\begin{array}{l}\text { Histopathology with } \\
\text { severe } \\
\text { immunosuppression }\end{array}$ & Specificity & $\begin{array}{l}0.12 \\
(0.03 \\
0.31)\end{array}$ & $\begin{array}{l}0.28(0.12, \\
0.49)\end{array}$ & $\begin{array}{l}0.68(0.46, \\
0.85)\end{array}$ & $0.60(0.39,0.79)$ \\
\hline & Sensitivity & $\begin{array}{l}0.96 \\
(0.78 \\
1.00)\end{array}$ & $\begin{array}{l}0.65(0.43, \\
0.84)\end{array}$ & $\begin{array}{l}0.30(0.13, \\
0.53)\end{array}$ & $0.52(0.31,0.73)$ \\
\hline
\end{tabular}

Specificity and sensitivity for patients with examination of lung tissue for histopathological examination. (95\% Confidence Interval).

\section{Subgroup and sensitivity analyses}

A subgroup analysis of severely immunocompromised patients $(n=256,37 \%)$ (defined as neutropenia, hematological malignancy, chemotherapy, stem cell transplantation, human immunodeficiency virus positivity, solid organ transplantation and/or immunosuppressive medication) revealed very poor agreement among the four diagnostic criteria (Additional File 2). Sensitivity and specificity for immunocompromised patients with lung tissue histopathology $(\mathrm{n}=48)$ are presented in Table 3. 
To further determine the diagnostic accuracy of the four diagnostic algorithms, we repeated the agreement analyses in a subgroup of patients without viral pneumonia (influenza and COVID-19; n=635). Cohen's kappa again revealed very poor agreement (Additional File 3). In addition, we determined the diagnostic accuracy of confirmed IPA for the subgroup of patients $(n=80)$ with lung tissue excluding patients with viral community-acquired pneumonia (influenza and SARS-CoV-2; Table 3).

\section{Discussion}

The main findings of our study were: 1) A comparison of four algorithms for the diagnosis of probable IPA in critically ill patients showed poor agreement among single diagnostic criteria. 2) In patients with proven IPA confirmed by lung tissue histopathology, the new EORTC/MSGERC ICU*2021 definition showed the highest diagnostic accuracy.

The diagnosis of IPA is not only complex, but is changing with new challenges in critical care medicine. Over the past years, there has been a rising incidence of IPA in ICUs, since more and more patients without traditional host-related risk factors have become susceptible resulting from advances in acute care and extracorporeal organ support $[1,16]$.

Diagnosis remains challenging, since proven IPA requires lung biopsy $[5,9,11,12]$ that can frequently not be performed in critically ill patients owing to coagulopathy or impaired gas exchange [10,11]. Timely diagnosis and accurate discrimination of infection from colonization is of utmost importance to allow for rapid treatment. At the same time, unnecessary antifungal medication in patients with Aspergillus colonization should be avoided due to its potential adverse effects, including antifungal resistance, drugdrug interactions, and economic impact [16].

In the absence of lung tissue for histopathological confirmation, the diagnosis of IPA is based on mycology, medical imaging, underlying conditions, and acute disease expression [17]. Over the past decades, the diagnostic spectrum has been enlarged allowing for less invasive tools to diagnose IPA. New diagnostic methods including assays for Aspergillus galactomannan antigen, lateral flow assays, and Aspergillus PCR were gradually integrated into the diagnostic algorithms [18-20]. Despite advances in diagnostic tools, underlying conditions and clinical signs frequently prompt the initiation of antifungal treatment [17].

To meet the specific demand of the critical care setting, the AspICU criteria have been developed in 2004 [21] and proposed in 2012 to discriminate IPA from Aspergillus colonization in non-neutropenic ICU patients. The clinical criteria of the AspICU algorithm [14,21] are not always easy to apply in critically ill patients, since neither dyspnoea, chest pain nor pleural rub may be detectable in patients with mechanical ventilation and sedation or fever may be masked by antipyretic intervention. Importantly, the radiological features that are limited to a pathological X-ray, apply to a substantial number of critically ill patients [22].

The influenza pandemic in 2009 particularly pronounced the need for alternative diagnostic criteria for IPA in non-neutropenic patients [23]. The differentiation between invasive fungal infection and Aspergillus colonization poses a major challenge in IPA diagnosis, particularly in non-neutropenic patients. As a 
consequence, Schauwvlieghe et al. proposed modified AspICU criteria in 2018 that allowed for the diagnosis of probable IPA in influenza patients and omitted all host factors [5].

Based on the modified AspICU criteria from 2018, a different approach was chosen by Loughlin et al., who focused on clinical signs and measurable parameters (body temperature $<35^{\circ} \mathrm{C}$ or $>38^{\circ} \mathrm{C}$, leukocytosis, leukopenia) [9]. Purulent tracheal secretion and new or increasing infiltrates on lung X-ray were considered as indicative for IPA [9]. Of all diagnostic criteria assessed, we found the highest specificity and lowest sensitivity in the modified AspICU criteria by Loughlin et al. from 2020. This is probably attributable to the unspecific clinical and radiological signs used that may be present in a high number of critically ill patients, regardless of the presence of IPA.

Recently, the EORTC/MSGERC ICU Working Group proposed a new definition of IPA in ICU patients [12]. As opposed to previous criteria, the new algorithm encompasses the Aspergillus galactomannan antigen assay, more distinct radiological characteristics, and new host factors including severe influenza and COVID19 [12]. Although far from optimal, we found both high specificity and high sensitivity to confirm proven IPA for the new criteria when compared to the remaining three algorithms included.

The 2018, 2020, and 2021 algorithms have been derived from the AspICU criteria proposed in $2012[5,9,12,14]$. Minor changes in the most recent algorithms result in poor agreement in the diagnosis of probable IPA as reflected by our results. Probable IPA as defined by all criteria was present in only $4 \%$ of study participants owing to changes in radiologic features required for diagnosis. While the early AspICU criteria encompassed any pathology on chest X-ray, the most recent EORTC/MSGERC ICU Working Group criteria require more distinct findings such as circumscribed lesions with or without halo signs, air crescent signs, cavities or wedge-shaped consolidation [12]. We observed a decrease in sensitivities and increasing specificity across the four algorithms. Our findings suggest that Aspergillus colonization is more likely to be recognized at the cost of more patients with IPA remaining untreated.

We performed a sensitivity analysis excluding patients with viral community-acquired pneumonia. The results from the sensitivity analysis confirmed poor agreement among the diagnostic algorithms. Consistent diagnostic criteria are required for the vast majority of critically ill patients. This has become even more apparent with the SARS-CoV-2 pandemic. There is an ongoing debate on the relevance of COVID-19associated pulmonary aspergillosis (CAPA) due to the heterogeneity in the reported incidence [24-26]. A retrospective analysis of 186 patients with CAPA also included patients with positive Aspergillus antigen tests from non-bronchoscopic lavage instead of the BAL [3]. Interestingly, a recent study showed that the incidence of probable IPA was significantly lower in the SARS-CoV-2 pneumonia group than in the influenza pneumonia group ( $2.5 \%$ vs. $6 \%$ ) [6]. However, the SARS-CoV-2 pandemic has pointed out the necessity for limiting aerosol-generating procedures. To meet the specific demands during the SARS-CoV-2 pandemic a diagnostic scheme that includes Aspergillus galactomannan testing in non-bronchoscopic lavage has already been proposed [27].

Strengths and limitations

Page 14/23 
Our retrospective analysis has several limitations that need to be addressed. The study population included a relatively low number of patients with lung tissue available for histopathological diagnostics. However, the paucity of biopsy material is one key limitation in the diagnosis of IPA in critically ill patients that underlines the importance of algorithms that rely on factors other than lung tissue. The modified AspICU* 2018 criteria were among the four algorithms that were compared in this study [5]. These criteria were primarily derived from the AspICU*2012 criteria to diagnose IPA in patients with influenza [5]. However, the authors applied their criteria to a cohort of critically ill patients not suffering from influenza. As a consequence, Loughlin et al. modified the AspICU*2018 criteria for application in a large cohort of critically ill patients $[9,13]$. Therefore, we deemed it appropriate to compare the AspICU*2018 criteria with other diagnostic algorithms. Of note, new diagnostic criteria for influenza-associated IPA have been proposed recently [13]. According to this definition all influenza patients in our cohort would have fulfilled the criteria for proven or probable IPA.

One strength of our study is the large cohort of 684 patients with positive Aspergillus culture in respiratory secretions. We analyzed data from a mixed population of critically ill patients including immunocompromised conditions and apparently immunocompetent subjects. To address the specific characteristics of the latter group we performed a sensitivity analysis that confirmed low agreement among the diagnostic criteria. Despite the single-center design, the heterogeneity of our study population may increase generalizability.

\section{Conclusion}

To the best of our knowledge this is the first study to compare four algorithms for the diagnosis of IPA in a cohort of mixed ICU patients. Agreement among the four algorithms was low, which underlines the major challenge of discriminating invasive fungal infection from colonization in critically ill patients. In a subgroup of patients with histopathological examinations, we found the highest specificity for the modified AspICU*2020 algorithm, the highest sensitivity for the AspICU*2012 algorithm and both high sensitivity and specificity for the new EORTC/MSGERC ICU Working Group criteria that have been proposed in March 2021 [12]. We therefore conclude that the new criteria sufficiently detect proven IPA. Yet, their ability to differentiate between probable IPA requiring antifungal treatment and colonization remains to be determined.

\section{Abbreviations}

APACHE II: $\quad$ Acute Physiology and Chronic Health Score

ARDS: $\quad$ Acute Respiratory Distress Syndrome

COVID-19: $\quad$ Coronavirus disease 2019

ECMO: $\quad$ Extracorporeal membrane oxygenation

EORTC: $\quad$ European Organisation for Research and Treatment of Cancer 
ICU: Intensive Care Unit

IPA: $\quad$ Invasive pulmonary aspergillosis

IQR: Interquartile range

LOS: $\quad$ Length of stay

MSGERC: $\quad$ Mycoses Study Group Education and Research Consortium

SAPS II: $\quad$ Simplified Acute Physiology Score II

SARS-CoV-2: $\quad$ Severe Acute Respiratory Syndrome Coronavirus Type 2

SOFA: $\quad$ Sequential Organ Failure Assessment Score

\section{Declarations}

\section{Consent to Publish}

Not Applicable - identifying images or other personal or clinical details of participants are not presented.

Availability of data and materials

The datasets used and/or analysed during the current study are available from the corresponding author on reasonable request.

\section{Competing interests}

MSch has received research support from Pfizer.

JG has received research support from Adroit Surgical, Ambu, ETView, Infectopharm, and Pfizer, and received consultant and lecture fees from Drägerwerk, Fresenius Medical, GE Healthcare, and Smith Medical, and holds shares from AstraZeneca, Bayer, Gilead, and Pfizer. D. W. reports personal consultant and lecture fees from AMEOS, Advanz (formerly Correvio), Eumedica, EUSA, Gilead, Kite, Lilly, MSD, Novartis, Pfizer, and Shionogi.

S K received research support from Cytosorbents and Daiichi Sankyo. He also received lecture fees from Astra, Bard, Baxter, Biotest, Cytosorbents, Daiichi Sankyo, Fresenius Medical Care, Gilead, Mitsubishi Tanabe Pharma, MSD, Pfizer, Philips and Zoll. He received consultant fees from Fresenius, Gilead, MSD and Pfizer.On behalf of all other authors, the corresponding author states that there are no competing interests.

\section{Funding}

Open Access funding enabled and organized by Pfizer $\mathrm{GmbH}$ supported this study with an IIR Grant. The company did not have any role in study design, data collection, data analysis, data interpretation, preparing the report or any decision contributing to its publication. MSch receives funding from the Medical Faculty of 
the University of Hamburg for clinical leave. MF receives research support from the Johanna und Fritz Buch Gedächtnis-Stiftung.

\section{Authors' contributions}

All authors have read and approved the manuscript.

MSch, MF, SK conception of the work, MG, CW, SW, AN, JG, HM acquisition of data, JG, MSch, MF, AO analysis of data, MSch, DW, MF, SK interpretation of data, HG, MC acquisition of microbiological data, MSch, AN, SS, AH acquisition of histological findings, MSch, MF draft the manuscript, DW, MS, JS, JG revision for important intellectual content.

\section{Acknowledgements}

We thank Sarah Minner and Malte Schroeder for support in this work.

\section{References}

1. Latgé JP, Chamilos G. Aspergillus fumigatus and Aspergillosis in 2019. Clin Microbiol Rev. $2019 ; 33$.

2. Koehler P, Cornely OA, Bottiger BW, Dusse F, Eichenauer DA, Fuchs F, et al. COVID-19 associated pulmonary aspergillosis. Mycoses [Internet]. 2020;63:528-34. Available from:

https://www.ncbi.nlm.nih.gov/pubmed/32339350

3. Salmanton-García J, Sprute R, Stemler J, Bartoletti M, Dupont D, Valerio M, et al. COVID-19-Associated Pulmonary Aspergillosis, March-August 2020. Emerg Infect Dis. 2021;27:1077-86.

4. Rutsaert L, Steinfort N, Hunsel TV, Bomans P, Naesens R, Mertes H, et al. COVID-19-associated invasive pulmonary aspergillosis. Ann Intensive Care. 2020;10:71.

5. Schauwvlieghe A, Rijnders BJA, Philips N, Verwijs R, Vanderbeke L, Tienen CV, et al. Invasive aspergillosis in patients admitted to the intensive care unit with severe influenza: a retrospective cohort study. Lancet Respir Med [Internet]. 2018;6:782-92. Available from:

https://www.ncbi.nlm.nih.gov/pubmed/30076119

6. Rouzé A, Lemaitre E, Martin-Loeches I, Povoa P, Diaz E, Nyga R, et al. Invasive pulmonary aspergillosis among intubated patients with SARS-CoV-2 or influenza pneumonia: a European multicenter comparative cohort study. Critical Care [Internet]. 2022;26:11. Available from: https://doi.org/10.1186/s13054-021-03874-1

7. Taccone FS, Abeele AMV den, Bulpa P, Misset B, Meersseman W, Cardoso T, et al. Epidemiology of invasive aspergillosis in critically ill patients: clinical presentation, underlying conditions, and outcomes. Crit Care [Internet]. 2015;19:7. Available from: https://www.ncbi.nlm.nih.gov/pubmed/25928694

8. Meersseman W, Lagrou K, Maertens J, Wijngaerden EV. Invasive aspergillosis in the intensive care unit. Clin Infect Dis [Internet]. 2007;45:205-16. Available from: https://www.ncbi.nlm.nih.gov/pubmed/17578780 
9. Loughlin L, Hellyer TP, White PL, McAuley DF, Morris AC, Posso RB, et al. Pulmonary Aspergillosis in Patients with Suspected Ventilator-associated Pneumonia in UK ICUs. Am J Respir Crit Care Med [Internet]. 2020;202:1125-32. Available from: https://www.ncbi.nlm.nih.gov/pubmed/32609533

10. Shen $\mathrm{H}$, Zhang Z, Yu Y. Diagnosis of invasive pulmonary aspergillosis in the intensive care unit: what we should concern and how to do better. Journal of Emergency and Critical Care Medicine [Internet]. 2019;3. Available from: https://jeccm.amegroups.com/article/view/5450

11. Tello K, Richter MJ, Seeger W, Hecker M. Nosokomial erworbene Pneumonie. Medizinische Klinik Intensivmedizin und Notfallmedizin [Internet]. 2018;113:685-95. Available from: https://doi.org/10.1007/s00063-018-0494-y

12. Bassetti M, Azoulay E, Kullberg BJ, Ruhnke M, Shoham S, Vazquez J, et al. EORTC/MSGERC Definitions of Invasive Fungal Diseases: Summary of Activities of the Intensive Care Unit Working Group. Clin Infect Dis [Internet]. 2021;72:S121-7. Available from:

https://www.ncbi.nlm.nih.gov/pubmed/33709127

13. Verweij PE, Rijnders BJA, Brüggemann RJM, Azoulay E, Bassetti M, Blot S, et al. Review of influenzaassociated pulmonary aspergillosis in ICU patients and proposal for a case definition: an expert opinion. Intensive Care Med. 2020;46:1524-35.

14. Blot SI, Taccone FS, Abeele AMV den, Bulpa P, Meersseman W, Brusselaers N, et al. A clinical algorithm to diagnose invasive pulmonary aspergillosis in critically ill patients. Am J Respir Crit Care Med [Internet]. 2012;186:56-64. Available from: https://www.ncbi.nlm.nih.gov/pubmed/22517788

15. Donnelly JP, Chen SC, Kauffman CA, Steinbach WJ, Baddley JW, Verweij PE, et al. Revision and Update of the Consensus Definitions of Invasive Fungal Disease From the European Organization for Research and Treatment of Cancer and the Mycoses Study Group Education and Research Consortium. Clin Infect Dis [Internet]. 2020;71:1367-76. Available from: https://www.ncbi.nlm.nih.gov/pubmed/31802125

16. Thompson GR, Young J-AH. Aspergillus Infections. New England Journal of Medicine [Internet]. 2021;385:1496-509. Available from: https://www.nejm.org/doi/full/10.1056/NEJMra2027424

17. Blot S, Rello J, Koulenti D. Diagnosing invasive pulmonary aspergillosis in ICU patients: putting the puzzle together. Curr Opin Crit Care. 2019;25:430-7.

18. Ullmann AJ, Aguado JM, Arikan-Akdagli S, Denning DW, Groll AH, Lagrou K, et al. Diagnosis and management of Aspergillus diseases: executive summary of the 2017 ESCMID-ECMM-ERS guideline. Clin Microbiol Infect [Internet]. 2018;24 Suppl 1:e1-38. Available from: https://www.ncbi.nlm.nih.gov/pubmed/29544767

19. Schroeder M, Simon M, Katchanov J, Wijaya C, Rohde H, Christner M, et al. Does galactomannan testing increase diagnostic accuracy for IPA in the ICU? A prospective observational study. Crit Care [Internet]. 2016;20:139. Available from: https://www.ncbi.nlm.nih.gov/pubmed/27160692

20. Prattes J, Flick H, Pruller F, Koidl C, Raggam RB, Palfner M, et al. Novel tests for diagnosis of invasive aspergillosis in patients with underlying respiratory diseases. Am J Respir Crit Care Med [Internet]. 2014;190:922-9. Available from: https://www.ncbi.nlm.nih.gov/pubmed/25203869 
21. Vandewoude K, Blot S, Benoit D, Depuydt P, Vogelaers D, Colardyn F. Invasive aspergillosis in critically ill patients: analysis of risk factors for acquisition and mortality. Acta Clin Belg [Internet]. 2004;59:251-7. Available from: https://www.ncbi.nlm.nih.gov/pubmed/15641394

22. Jenks JD, Nam HH, Hoenigl M. Invasive aspergillosis in critically ill patients: Review of definitions and diagnostic approaches. Mycoses. 2021;64:1002-14.

23. Vanderbeke L, Spriet I, Breynaert C, Rijnders BJA, Verweij PE, Wauters J. Invasive pulmonary aspergillosis complicating severe influenza: epidemiology, diagnosis and treatment. Curr Opin Infect Dis. 2018;31:471-80.

24. Fekkar A, Lampros A, Mayaux J, Poignon C, Demeret S, Constantin JM, et al. Occurrence of Invasive Pulmonary Fungal Infections in Patients with Severe COVID-19 Admitted to the ICU. Am J Respir Crit Care Med. 2021;203:307-17.

25. Lahmer T, Kriescher S, Herner A, Rothe K, Spinner CD, Schneider J, et al. Invasive pulmonary aspergillosis in critically ill patients with severe COVID-19 pneumonia: Results from the prospective AspCOVID-19 study. PLOS ONE [Internet]. 2021;16:e0238825. Available from: https://doi.org/10.1371/journal.pone.0238825

26. Paparoupa M, Aldemyati R, Roggenkamp H, Berinson B, Nörz D, Olearo F, et al. The prevalence of earlyand late-onset bacterial, viral, and fungal respiratory superinfections in invasively ventilated COVID-19 patients. J Med Virol. 2022;

27. Koehler P, Bassetti M, Chakrabarti A, Chen SCA, Colombo AL, Hoenigl M, et al. Defining and managing COVID-19-associated pulmonary aspergillosis: the 2020 ECMM/ISHAM consensus criteria for research and clinical guidance. Lancet Infect Dis [Internet]. 2021;21:e149-62. Available from: https://www.ncbi.nlm.nih.gov/pubmed/33333012

\section{Figures}




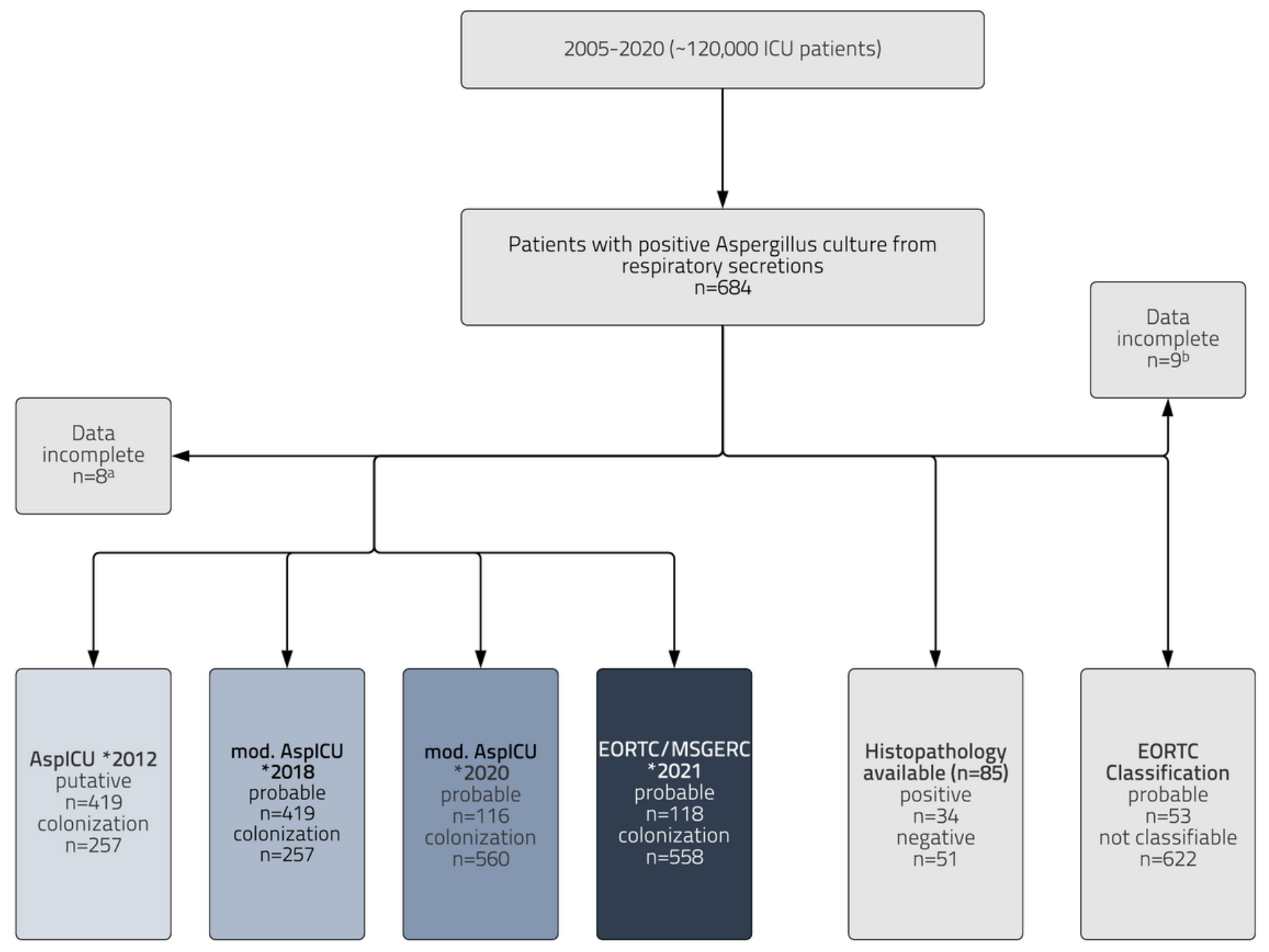

Figure 1

Flow of participants throughout the study period from 2005 to 2020.

AspICU*2012 [14], modified AspICU*2018 [5], modified AspICU*2020 [9], EORTC/MSGERC ICU*2021[12], EORTC Classification [15]

EORTC/MSGERC: European Organisation for Research and Treatment of Cancer Mycoses Study Group Education and Research Consortium. ICU: intensive care unit. 


\section{Venn diagram for positive probable diagnosis}

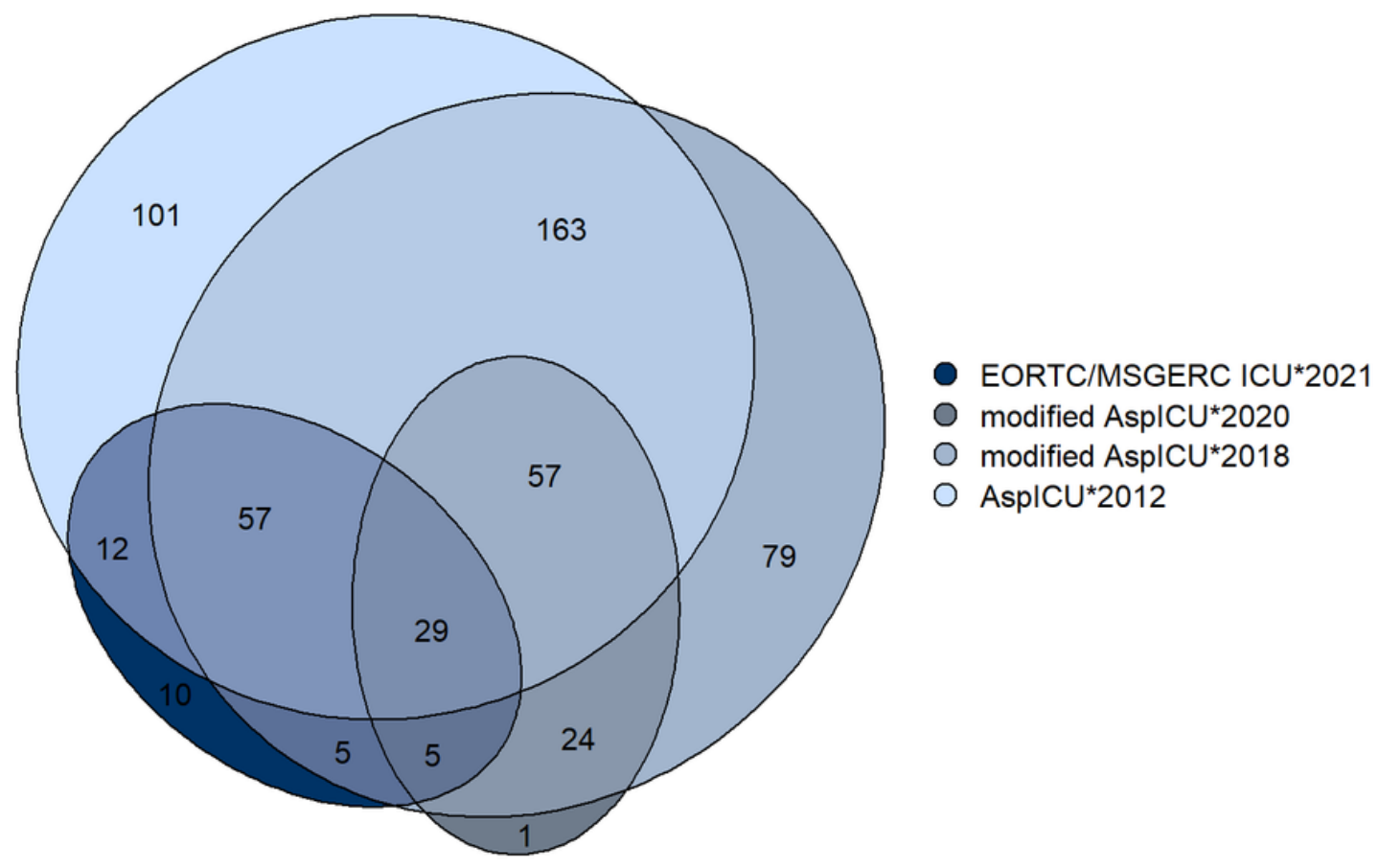

Figure 2

Venn diagram for the diagnosis of probable invasive pulmonary Aspergillosis.

Number of patients, who fulfill the criteria for probable invasive pulmonary aspergillosis according to one or more of four diagnostic algorithms $[5,9,12,14]$. 
A

AsplCU*2012 vs Modified AsplCU*2018

Cohens kappa (95\%Cl): $0.29(0.22,0.37)$

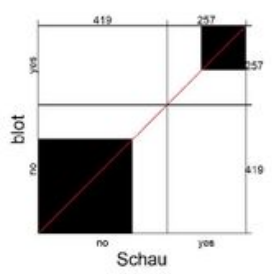

C

AspICU*2012 vs EORTC/MSGERG ICU*2021 Cohens kappa (95\%Cl): $0.13(0.06,0.19)$

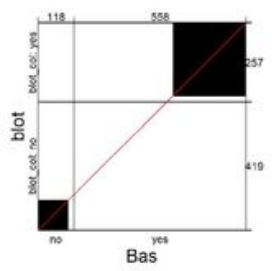

E

Modified AspICU*2018 vs EORTC/MSGERG ICU*2021 Cohens kappa (95\%Cl): $0.12(0.05,0.18)$

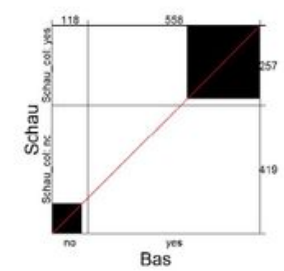

B

AsplCU*2012 vs Modified AsplCU*2020

Cohens kappa (95\%Cl): $0.07(0.01,0.14)$

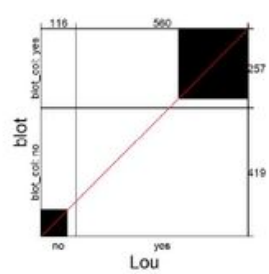

D

Modified AsplCU*2018 vs Modified AsplCU*2020 Cohens kappa (95\%CI): $0.22(0.16,0.29)$

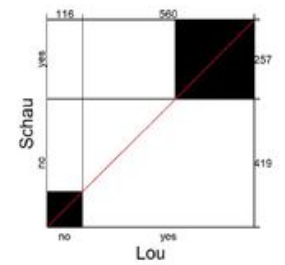

Modified AspICU*2020 vs EORTC/MSGERG ICU*2021 Cohens kappa $(95 \% \mathrm{Cl}): 0.14(0.03,0.26)$

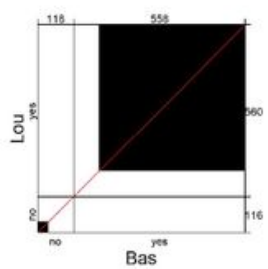

\section{Figure 3}

Agreement among four algorithms for the diagnosis of probable invasive pulmonary Aspergillosis.

A-F: Comparison of four algorithms $[5,9,12,14]$ among each other, expressed by agreement plots and Cohen's Kappa with 95\% confidence interval. 


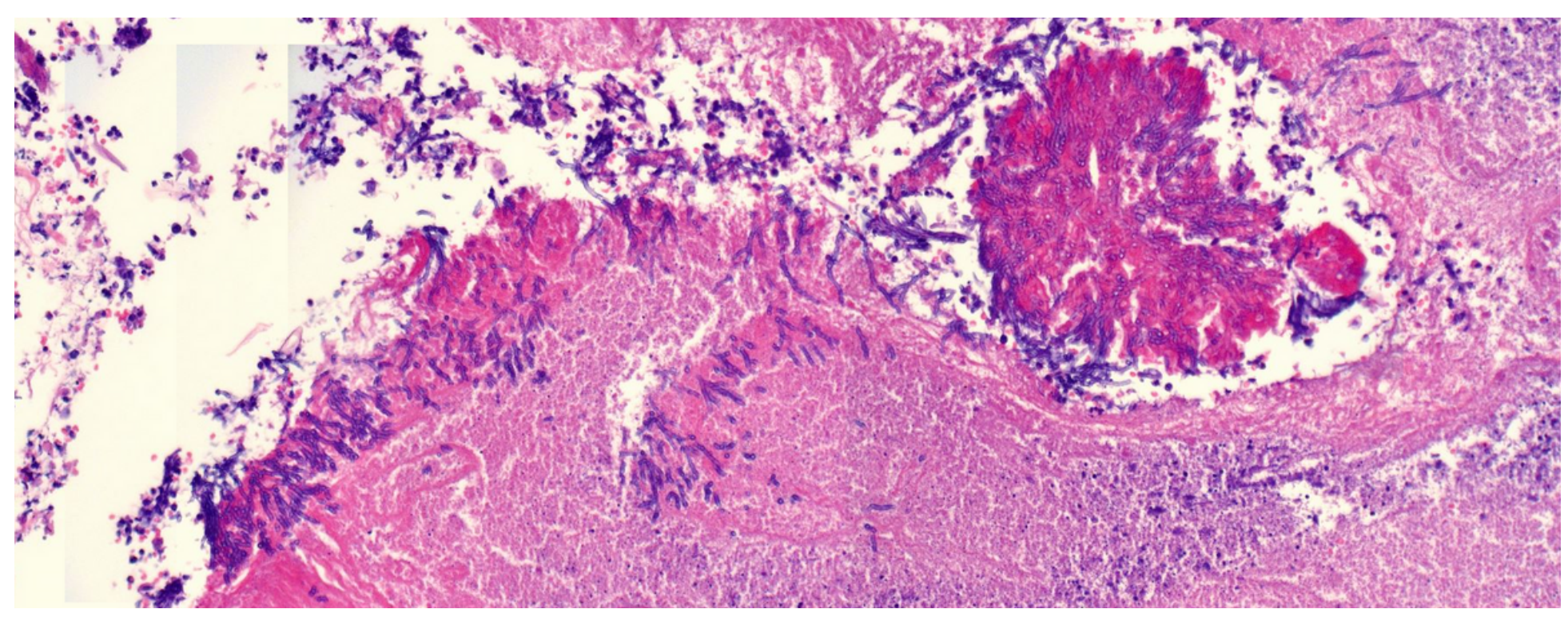

Figure 4

Example of one histopathological examination of lung tissue with invasive pulmonary aspergillosis.

Patient \#512, male, died 21 days after the first positive culture for Aspergillus fumigatus. Histological examination of a biopsy specimen obtained during postmortem examination; Aspergillus spp. in the center of a destroyed bronchus; HE-stain, 20x, fungi hyphae are violet.

\section{Supplementary Files}

This is a list of supplementary files associated with this preprint. Click to download.

- Additionalfiles.pdf

- GraphicalAbstract.png 